\title{
FIGO Stage IB3
}

National Cancer Institute

\section{Source}

National Cancer Institute. FIGO Stage IB3. NCI Thesaurus. Code C162222.

A FIGO stage term that applies to gynecologic cancers. For cervical cancer, it refers to invasive cancer measuring $4 \mathrm{~cm}$ or more in greatest dimension. 